\title{
Direct identification of As vacancies in GaAs using positron annihilation calibrated by scanning tunneling microscopy
}

\author{
J. Gebauer and R. Krause-Rehberg \\ Fachbereich Physik, Martin-Luther-Universität Halle-Wittenberg, D-06099 Halle, Germany \\ C. Domke, Ph. Ebert, and K. Urban \\ Institut für Festkörperforschung, Forschungszentrum Jülich GmbH, D-52425 Jülich, Germany \\ T. E. M. Staab \\ Laboratory of Physics, Helsinki University of Technology, P.O. Box 1100, FIN-02015 HUT, Finland
}

(Received 16 May 2000; revised manuscript received 1 August 2000; published 8 January 2001)

\begin{abstract}
We report on the identification of native vacancies in GaAs by positron annihilation with a special emphasis on As vacancy-related defects. In annealed highly Si-doped GaAs, we observe a neutral vacancy defect with a positron lifetime $\tau$ of $280-285$ ps and a high intensity of the core annihilation, in contrast to Ga vacancies which exhibit a lifetime of $\sim 260 \mathrm{ps}$ and a lower intensity of the core annihilation. This defect is identified by scanning tunneling microscopy measurements to be an As vacancy $\mathrm{Si}_{\mathrm{Ga}}$ donor complex. We find that the same defect is also present in low $n$-doped GaAs, where it was earlier assigned to a neutral As vacancy. The high positron lifetime is explained by a large outward lattice relaxation. Theoretical calculations of the momentum distribution employing free atomic wave functions are in good agreement with the experimental results, provided only relative changes are considered and an outward lattice relaxation is included which yields the experimental positron lifetime. These calculations also yield annihilation parameters for Ga vacancies, in good agreement with the experiment. Our results demonstrate that vacancies in both sublattices of GaAs can directly and unambiguously be identified by positron annihilation once the annihilation characteristics are calibrated by a complementary method. On the basis of this identification, the abundance of As vacancies in GaAs is discussed in terms of stoichiometry and formation energies.
\end{abstract}

DOI: 10.1103/PhysRevB.63.045203

PACS number(s): 78.70.Bj, 61.72.Ji, 68.37.Ef

\section{INTRODUCTION}

Defects govern to a large degree the properties of semiconductors. In order to predict the effect of a particular ensemble of defects on the electronic or optical properties of a semiconductor, it is necessary to unravel not only the properties of the defects, but also to identify their types and concentrations. Positron annihilation spectroscopy (PAS) has proven to be a very versatile and useful tool to determine in particular the concentrations and nature of vacancy defects. ${ }^{1}$ However, an unambiguous identification of the vacancy which traps the positron often remained a difficult problem in compound semiconductors, where anion and cation vacancies and complexes between these vacancies and dopant atoms can occur. This is a particular important problem in GaAs, since the electronic structures of Ga and As are very similar, and, hence, should lead to nearly the same positron lifetime for vacancies on both sublattices.

In order to overcome this drawback, the method of Doppler coincidence spectroscopy was recently developed especially for defect identification in semiconductors, ${ }^{2-4}$ extending earlier work on metals. ${ }^{5,6}$ This technique allows one to probe the annihilation momentum distribution at high momentum, where it carries chemical information due to annihilation with core electrons. ${ }^{2,4}$ This effect can be used to identify the chemical surrounding of vacancies, i.e., the sublattice, and formation of impurity vacancy complexes. It is possible to calculate the core annihilation with rather simple methods based on free atomic wave functions. Such calcula- tions in many cases allow an unambiguous identification of vacancy defects because the momentum distribution for several defect configurations can be efficiently computed and compared with experimental results. ${ }^{2}$ This was demonstrated, e.g., for $V_{\mathrm{Ga}}-\mathrm{Te}_{\mathrm{As}}$ complexes in Te-doped GaAs. ${ }^{7}$ However, the theoretical description has some limitations which hinder a detailed defect identification in certain cases: For example, the core annihilation in GaAs is dominated by $3 d$ electrons, which carry high momenta but which are less localized in GaAs than in the free atoms. ${ }^{3}$ As a result, calculations based on the wave functions of free atoms yield intensities of the core annihilation that are too high, especially with $\mathrm{Ga} 3 d$ electrons. Since the momentum distribution at As vacancies is dominated by annihilation with $3 d$ electrons from the surrounding $\mathrm{Ga}$ atoms, it was assumed that such calculations would not yield reliable results, i.e., a too high intensity for the momentum distribution at As vacancies. ${ }^{3}$ It is possible to improve the calculation of annihilation parameters by using more sophisticated theoretical methods, including the effect of the true band structure of the solid. ${ }^{8}$ However, the improved calculations are computationally much more demanding than simpler ones based on atomic wave functions. A direct identification of As vacancies in GaAs by PAS has thus not yet been achieved. Earlier assignments of defects to As vacancies were based on indirect arguments, i.e., on an interpretation of ionization levels. ${ }^{9-12}$ The momentum distribution of such vacancies was characterized by angular correlation of annihilation radiation, ${ }^{13,14}$ which mainly probes the low-momentum range and thus pro- 
vides no direct chemical sensitivity. An independent and direct defect identification was not attempted, as comparison with theory was limited to the simple case of isolated vacancies and yielded only a qualitative agreement. ${ }^{15}$ The assignment to As vacancies was therefore based on an earlier interpretation of ionization levels. ${ }^{9-12}$

It is, however, desirable to obtain a direct microscopic defect identification using PAS for all types of vacancies. This direct identification can be achieved by calibrating the PAS signal using a reference standard, i.e., a sample known to contain As vacancies. The experimental results could then be compared to theoretical calculations, which would in turn allow one to test the degree to which the approximations in the calculation continue to yield accurate results. Ultimately, such a calibrated PAS would allow one to provide, for samples with unknown types of vacancy defects, a full identification of the vacancy defects present. The methodology outlined above depends on the availability of suitable reference samples. For providing such reference samples it is necessary to identify the presence of As vacancies in GaAs on the basis of a complementary method. This can be achieved by cross-sectional scanning tunneling microscopy (STM) ${ }^{16}$ where the bulk defects are exposed on a cleavage surface and directly imaged with atomic resolution. Atomically resolved STM images provide the basis for an identification of the defects, and enable measurements of their concentration. ${ }^{17,18}$ Since such experiments are rather complex and time consuming, STM can only be applied to a few reference samples, which may then serve as a calibration standard for other methods like positron annihilation.

In the present work, we demonstrate the calibration of positron annihilation spectra of vacancies in GaAs using reference samples, whose defects were previously characterized by scanning tunneling microscopy. ${ }^{16,19}$ We identify the features in spectra, distinguishing positron annihilation in As vacancies from that in $\mathrm{Ga}$ vacancies by using reference samples with As vacancies. Furthermore, we test the extent to which calculations allow an accurate description of the spectra using the example of As vacancy-related complexes. Our results provide a methodology on how to identify unambiguously defects in both sublattices of GaAs by positron annihilation.

This paper is organized as follows. Section II describes the experimental details and sample materials investigated. In Sec. III A we demonstrate the detection of As vacancyrelated defects by PAS in low $n$-doped and annealed highly $\mathrm{Si}$-doped GaAs. These vacancies are shown to be complexes with $\mathrm{Si}_{\mathrm{Ga}}$ donors by STM in Sec. III B. In Sec. IV A annihilation parameters from theoretical calculations are shown to be in surprisingly good agreement with the experimental results, provided relative changes of the momentum distribution are considered. Finally, we discuss the role and abundance of $V_{\mathrm{As}}$ in light of earlier experimental and theoretical results.

\section{EXPERIMENT}

The samples investigated were Si-doped and intentionally undoped $n$-type GaAs. Highly Si-doped GaAs ([Si]
$=(2.5-6.0) \times 10^{19} \mathrm{~cm}^{-3}$, measured by secondary ion mass spectroscopy) was grown by the vertical Bridgman (VB) method. The samples were investigated in the as-grown state and after annealing for $2 \mathrm{~h}$ at $1130^{\circ} \mathrm{C}$ in a closed quartz glass ampoule under low As pressure. The carrier concentrations were $(1.2-2.0) \times 10^{18} \mathrm{~cm}^{-3}$ and $(4.5-7.0) \times 10^{18} \mathrm{~cm}^{-3}$ in asgrown and annealed materials, respectively. As a typical example for material previously thought to contain As vacancies $^{9-12}$ we investigate intentionally undoped, $n$-type GaAs grown by the horizontal Bridgman (HB) method. ${ }^{11}$ The carrier concentration was $n=5.9 \times 10^{16} \mathrm{~cm}^{-3}$. In this sample, a surprisingly high silicon concentration of 2.8 $\times 10^{18} \mathrm{Si} \mathrm{cm}^{-3}$ was detected by SIMS. For comparison, we investigate lightly Si-doped GaAs $\left(n=1 \times 10^{16} \mathrm{~cm}^{-3}\right)$ grown by the liquid-encapsulated Czochralski (LEC) method. Zndoped, LEC-grown GaAs $\left(p=5 \times 10^{17} \mathrm{~cm}^{-3}\right)$ was used as a reference for positron annihilation in GaAs free from positron trapping at vacancies.

Positron lifetime spectroscopy was performed using a conventional fast-fast-system (time resolution $250 \mathrm{ps}$ ) in a temperature range between 30 and $550 \mathrm{~K}$. About (4-6) $\times 10^{6}$ events were collected in each lifetime spectrum. The annihilation momentum distribution was observed by Doppler broadening coincidence spectroscopy at room temperature using a setup of two Ge detectors (system resolution $1.03 \mathrm{keV}) .{ }^{20}$ In each Doppler spectrum, about $1.5 \times 10^{7} \mathrm{co}-$ incident events were collected. The intensity of the annihilation with high-momentum core electrons was characterized by the $W$ parameter, defined as the relative intensity in the momentum range $(15-20) \times 10^{-3} m_{0} c$. The $W$ parameter is normalized to the value $W=0.0074$ of a GaAs: $Z n$ reference. Theoretical calculations of the annihilation parameters were performed employing free atomic wave functions using the method introduced in Refs. 2 and 3. The momentum distribution was obtained by summing up the contributions from each core-electron state weighted with its partial annihilation rate. The partial annihilation rate was calculated using the generalized gradient approximation (GGA) of electronpositron correlation. ${ }^{21}$ From the theoretical calculated momentum distribution, the $W$ parameter was determined in the same momentum range as the experimental one. Positron lifetimes were also calculated using the GGA scheme.

The highly Si-doped GaAs samples were investigated by STM in the as-grown state and after annealing. The samples were cleaved along a (110) plane in ultrahigh vacuum (5 $\times 10^{-9} \mathrm{~Pa}$ ), and investigated by scanning tunneling microscopy to observe the exposed bulk defects. Details of the technique used are described in Refs. 16 and 18.

\section{EXPERIMENTAL RESULTS}

\section{A. Defect assignment by positron annihilation}

Figure 1 shows the positron lifetime as a function of temperature of the different $n$-doped GaAs samples investigated. For comparison we included the respective data of $\mathrm{Zn}$-doped $\mathrm{GaAs}$, in which no vacancy defects are detected. The slight increase of the average positron lifetime $\tau_{\mathrm{av}}$ with temperature in $\mathrm{Zn}$-doped GaAs is due to thermal lattice expansion. ${ }^{1,12} \tau_{\mathrm{av}}$ is $228 \pm 1 \mathrm{ps}$ at $300 \mathrm{~K}$, in agreement with previous results for 


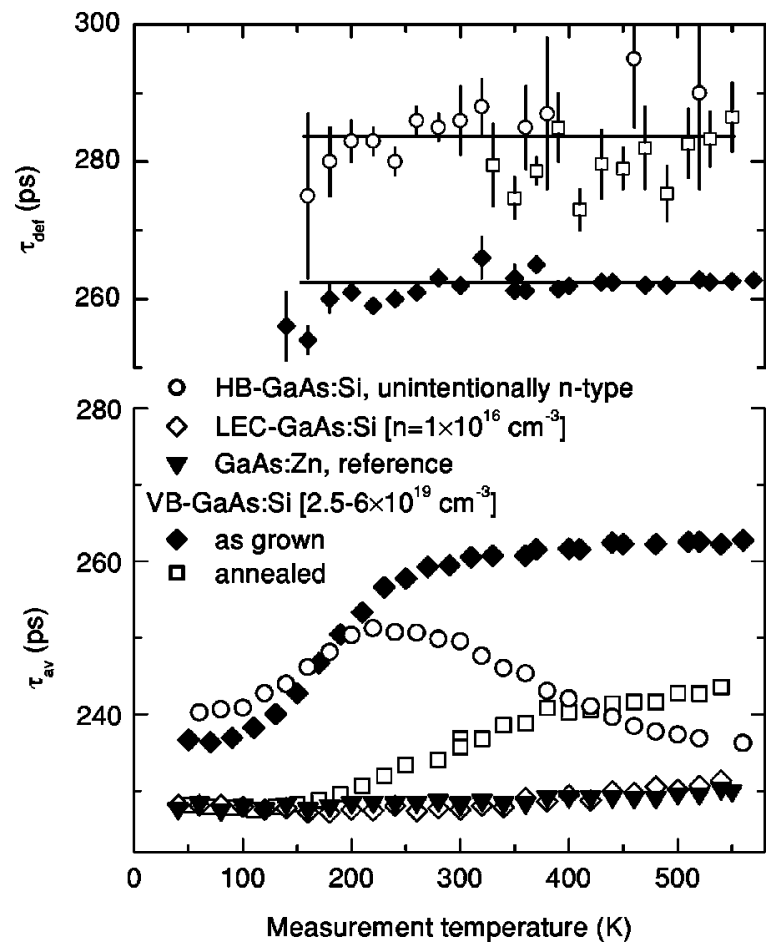

FIG. 1. Average positron lifetime (lower part) and defect-related lifetime (upper part) as a function of the temperature in different GaAs samples as indicated in the figure.

the positron lifetime $\tau_{b}$ in GaAs free from trapping at vacancies. Concentrating on the different $n$-doped samples we find rather large differences between them.

(i) As-grown highly $\mathrm{Si}$-doped GaAs has an average positron lifetime well above that of the bulk GaAs, indicating the presence of vacancy defects. We find a defect-related lifetime $\tau_{\text {def }}$ of $262 \pm 2$ ps. This result is in agreement with previous PAS and STM measurements of as-grown highly Sidoped GaAs. ${ }^{16,17}$ The vacancies were identified to be Gavacancy- $\mathrm{Si}_{\mathrm{Ga}}$-donor complexes with a concentration of 6 $\times 10^{18} \mathrm{~cm}^{-3}$. 17 The strong decrease of $\tau_{\mathrm{av}}$ to low temperatures is due to positron trapping at $\mathrm{Si}_{\mathrm{As}}{ }^{-}$acceptors. The $\mathrm{Si}_{\mathrm{As}}{ }^{-}$acceptors act as shallow positron traps at low temperatures with a positron lifetime close to the lifetime $\tau_{b}$ in the perfect GaAs lattice. ${ }^{1,22}$

(ii) Annealed highly Si-doped GaAs: The positron signal is different in a sample from the same highly Si-doped GaAs crystal after annealing: $\tau_{\mathrm{av}}$ is reduced and reaches $\tau_{b}$ at $T$ $<150 \mathrm{~K}$. $\tau_{\text {av }}$ is still above $\tau_{b}$ at high temperatures. Again this indicates the presence of vacancies. However, the defectrelated positron lifetime $\tau_{\text {def }}$ has increased to $280-285$ ps. This indicates a different nature, i.e., a larger open volume, of the vacancy defect compared to $V_{\mathrm{Ga}}-\mathrm{Si}_{\mathrm{Ga}}$ in as-grown material. On the other hand, $\tau_{\text {def }}$ is well below the value calculated for divacancies in GaAs (e.g., 332 ps in Ref. 7). Therefore, the vacancies in annealed highly $\mathrm{Si}$-doped GaAs should also be monovacancies. The decrease of the average positron lifetime $\tau_{\mathrm{av}}$ with temperature in annealed GaAs:Si is again attributed to positron trapping at negative ions, presumably $\mathrm{Si}_{\mathrm{As}}{ }^{-}$acceptors. The behavior of $\tau_{\mathrm{av}}$ as a function of temperature strongly indicates that the vacancies in annealed highly Si-doped GaAs are neutral. Positron trapping at neutral vacancies is independent of the temperature, whereas competing trapping at negative ions increases with decreasing temperature. ${ }^{23}$ Thus, in the case of neutral vacancies, $\tau_{\mathrm{av}}$ decreases permanently with decreasing temperature until either the lowest temperature or the value for annihilation at the negative ions (i.e., $\tau_{b}$ ) is reached. This is observed in Fig. 1 , showing that the vacancies are most probable neutral. For the determination of the vacancy concentration, one has to take into account that the trapping coefficient for neutral vacancies (in the low $10^{14}-\mathrm{s}^{-1}$ range) is known with limited accuracy only (for negative vacancies it is $10^{15} \mathrm{~s}^{-1}$ ). ${ }^{24}$ In addition, the negative ions influence positron trapping even at the highest measurement temperatures $\left(\tau_{\mathrm{av}}\right.$ does not reach a constant value). Therefore, we can only determine a lower limit of the vacancy concentration of about $5 \times 10^{17} \mathrm{~cm}^{-3}$ in annealed highly Si-doped GaAs.

(iii) The very low Si-doped GaAs sample has an average positron lifetime very similar to that of the $\mathrm{Zn}$-doped GaAs. This indicates a concentration of vacancies below the detection limit of about $5 \times 10^{15} \mathrm{~cm}^{-3}{ }^{24}$

(iv) A defect-related positron lifetime of $280-285 \mathrm{ps}$ is also found in the unintentionally $n$-doped GaAs sample. $\tau_{\mathrm{av}}$ is larger than $\tau_{b}$, but has a more complex dependence on temperature than in the other samples. The decrease of $\tau_{\mathrm{av}}$ at $T<150 \mathrm{~K}$ is attributed to positron trapping at negative ions as in the other samples. The increase of $\tau_{\mathrm{av}}$ with decreasing temperature between 200 and $500 \mathrm{~K}$ is, however, interpreted as the signature of a change of the charge state of the vacancies due to a temperature driven shift of the Fermi level toward the conduction band. ${ }^{11}$ The existence of an ionization level was previously confirmed with Hall-effect measurements. ${ }^{11}$ On this basis the vacancies in unintentionally doped GaAs were assigned to be As vacancies ${ }^{9-12}$ because the ionization levels of $V_{\mathrm{As}}$ are in the upper half of the band gap according to theory, whereas $\mathrm{Ga}$ vacancies have ionization levels below midgap. ${ }^{25,26}$ This conclusion is, however, crucially dependent on the accuracy of the calculated ionization levels, which can deviate rather significantly depending on the calculational method. Independent from that interpretation, we will characterize the vacancy defects in more detail. First, we found a defect-related positron lifetime $\tau_{\text {def }}$ of about $280-285 \mathrm{ps}$, very similar to that found in annealed highly Si-doped VB GaAs (see the upper part of Fig. 1). This indicates a similar type of vacancy defect in both materials. Bearing in mind the uncertainties of the trapping coefficient discussed above, we estimate a vacancy concentration of about $9 \times 10^{17} \mathrm{~cm}^{-3}$ in the unintentionally $n$-doped sample.

At this stage we have to apply Doppler broadening spectroscopy, since it provides additional information about the type of the vacancy present in the annealed highly Si-doped GaAs and the unintentionally $n$-doped GaAs. The fraction of positrons annihilating in vacancies is given by $\eta=\left(W_{b}\right.$ $-W) /\left(W_{b}-W_{\text {def }}\right)=\left(\tau_{\mathrm{av}}-\tau_{b}\right) /\left(\tau_{\mathrm{def}}-\tau_{b}\right)$. The measured $W$ parameter thus depends linearly on $\tau_{\mathrm{av}}$ if the defect density changes $(\eta)$ and not the defect type $\left(\tau_{\text {def }}\right.$ or $\left.W_{\text {def }}\right)$. Different linear variations are expected for different types of defects because $\tau_{\text {def }}$ or $W_{\text {def }}$ should be different. In Fig. $2, W$ is 


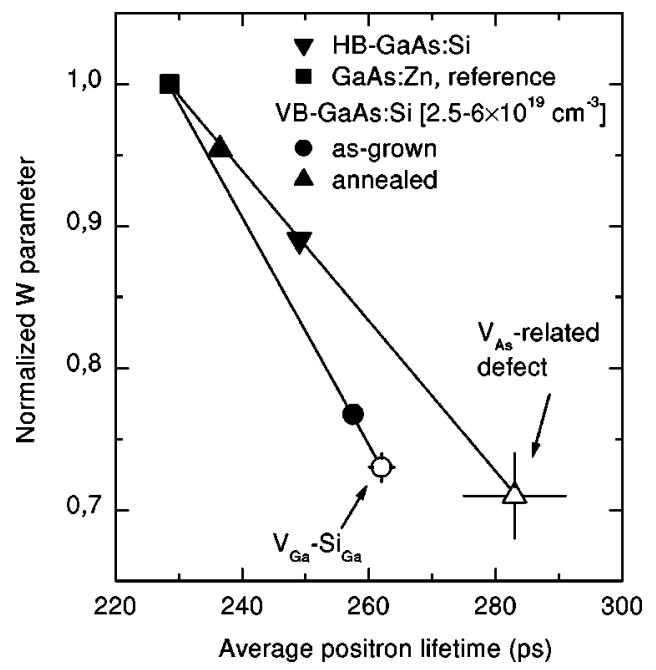

FIG. 2. $W$ parameter as a function of the average positron lifetime. Experimental data for the samples of this work are indicated by solid symbols. The individual errors for each data point are of the order of the symbol size. The annihilation parameters for the respective type of vacancies according to the trapping model, i.e., corresponding to full trapping, are indicated by open symbols.

shown as a function of $\tau_{\text {av }}$ for the samples investigated in this work. The data for the annealed highly Si-doped VB GaAs sample and the unintentionally $n$-type HB GaAs sample fall on the same line. Because we also detect the same defect-related lifetime, the vacancy defect is indeed the same in both samples. The linear variation is different from that in as-grown material, which is typical for $V_{\mathrm{Ga}}-\mathrm{Si}_{\mathrm{Ga}}$ complexes. ${ }^{7}$ Note that, for a given $\tau_{\mathrm{av}}$, the $W$ parameter for $V_{\mathrm{Ga}}-\mathrm{Si}_{\mathrm{Ga}}$ complexes is lower than that for the vacancy defects with a defect-related positron lifetime of 280-285 ps (Fig. 2). Thus the intensity of annihilation with highmomentum core electrons in the range $(15-20) m_{0} c$ is lower in $V_{\mathrm{Ga}}-\mathrm{Si}_{\mathrm{Ga}}$ complexes than in the vacancy defects with $\tau_{\text {def }}=280-285$ ps. In GaAs, the main contribution to the core annihilation comes from $3 d$ electrons. ${ }^{2,3}$ The $3 d$ electrons of $\mathrm{Ga}(Z=31)$ are more weakly bound than those of As $(Z=33)$, and thus have a larger spatial distribution. Positron annihilation in a vacancy occurs mainly with electrons belonging to the surrounding atoms. Therefore, the overlap with the positron wave function is stronger in the As vacancy surrounded by Ga atoms than in the Ga vacancy surrounded by As atoms. Thus the intensity of the annihilation with core electrons is expected to be larger for As vacancies compared to Ga vacancies. However, no influence or only a marginal influence on the positron lifetime is expected. ${ }^{2,3}$ It follows that the vacancies with $\tau_{\text {def }}=280-285$ ps are likely related to As vacancies because the intensity of the annihilation with high momentum core electrons is higher than that of $V_{\mathrm{Ga}}-\mathrm{Si}_{\mathrm{Ga}}$ complexes.

Additional support for this assignment comes from the detailed investigation of the annihilation momentum distribution (Fig. 3). The momentum distribution in annealed highly Si-doped GaAs and unintentionally $n$-doped GaAs decays steeper than the momentum distribution in as-grown highly Si-doped GaAs [Fig. 3(a)]. In order to reveal the dif-

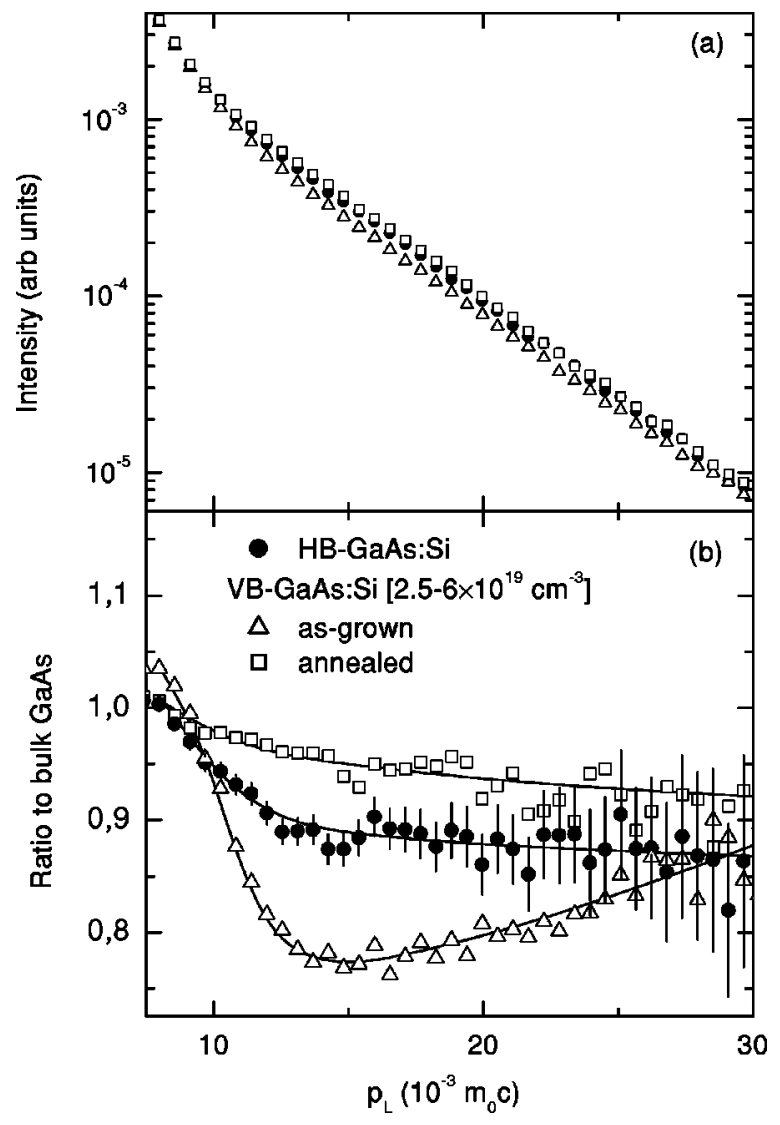

FIG. 3. (a) High-momentum part of the annihilation momentum distribution in annealed highly Si-doped VB GaAs ( $\square$ ), unintentionally $n$-doped HB GaAs $(\mathbf{O})$ and in as-grown highly Si-doped VB GaAs $(\triangle)$. The spectra are as measured. (b) High-momentum distribution for the same samples normalized to the data of a $\mathrm{Zn}$ doped GaAs reference sample. Symbols are the same as in (a). Lines are to guide the eye only.

ferences more clearly, in Fig. 3(b) we present the same data normalized to the data of the $\mathrm{Zn}$-doped GaAs reference sample. We show only the data at high momenta which are relevant to the annihilation with core electrons of interest here. ${ }^{3}$ Positron trapping at vacancies causes a decreased intensity of the core annihilation, whereas the annihilation with low-momentum valence electrons increases. For the normalized data, the intensity in the high-momentum region is therefore reduced compared to bulk GaAs (which corresponds to a ratio of 1).

The momentum distribution in as-grown highly Si-doped GaAs decays less steeper than in bulk GaAs, i.e., the ratio in Fig. 3(b) increases to high momentum. Core annihilation in bulk GaAs occurs with electrons from As and Ga. The more strongly bound As $3 \mathrm{~d}$ electrons are less localized in momentum space than the more weakly bound $\mathrm{Ga} 3 \mathrm{~d}$ electrons, i.e., the momentum distribution decays less steeper for annihilation with As $3 \mathrm{~d}$ electrons. ${ }^{3}$ The momentum distribution in as-grown highly Si-doped VB GaAs is therefore consistent with positron annihilation in Ga vacancies. ${ }^{7}$ The momentum distribution for isolated $V_{\mathrm{Ga}}$ has the same shape as the momentum distribution of $V_{\mathrm{Ga}^{-}} \mathrm{Si}_{\mathrm{Ga}}$, because the $\mathrm{Si}$ donor on the second next lattice site does not contribute significantly 
to the annihilation. ${ }^{7}$ In a recent study, we measured the annihilation momentum distribution for pure metallic As and As precipitates in nonstoichiometric GaAs layers. ${ }^{27}$ The shape of the momentum distribution was in agreement with that for $V_{\mathrm{Ga}}-\mathrm{Si}_{\mathrm{Ga}}$ as shown in Fig. 3, supporting our conclusions. The positron lifetime for annihilation at the As precipitates was about $345 \mathrm{ps},{ }^{27}$ ruling them out as being responsible for positron trapping in our bulk samples. This is in accordance with the knowledge that As precipitates in bulk GaAs have a concentration below the detection limit of positron annihilation. ${ }^{28}$ In contrast to the behavior discussed above, the momentum distribution in $n$-type HB GaAs and annealed highly Si-doped VB GaAs decays more steeply than in the bulk, i.e., the ratio decreases to high momenta. This indicates annihilation with weakly bound $\mathrm{Ga} 3 \mathrm{~d}$ electrons. Therefore, the vacancies should indeed be As vacancies, in agreement with the suggestion above. The shape of the momentum distribution is the same for both samples, again confirming independently that the defect type is the same in both samples.

To summarize the experimental findings of PAS, we detect in annealed and unintentionally $n$-doped GaAs vacancies with annihilation parameters different from that of $V_{\mathrm{Ga}}-\mathrm{Si}_{\mathrm{Ga}}$ complexes in as-grown highly Si-doped VB GaAs. The high intensity and the shape of the annihilation momentum distribution point toward As vacancies. However, a closer identification is not possible without additional information, i.e., it is not possible to decide whether the As vacancies are isolated or part of a defect complex. In principle, a comparison with theoretical calculations of the annihilation parameters could provide this information. However, at present it is not clear whether the calculations provide correct results for As vacancies as discussed above. Therefore, in Sec. III B we introduce reference samples characterized by scanning tunneling microscopy which allow us to relate the positron annihilation signals with a particular defect.

\section{B. Scanning tunneling microscopy of $V_{\mathrm{As}}-\mathrm{Si}_{\mathrm{Ga}}$ complexes}

We investigated a set of reference samples by STM to determine what kind of vacancies occur in these materials. For this purpose we exposed the defects by cleavage on a (110) cleavage surface, and imaged them by STM with atomic resolution. The nonpolar (110) cleavage surfaces of III-V semiconductors are well suited for the identification of point defects, due to their simple $1 \times 1$ reconstruction and the absence of surface states in the band gap. The observation of point defects by STM includes vacancies ${ }^{29,30}$ and vacancy complexes ${ }^{31,32}$ as well as bulk $\mathrm{As}_{\mathrm{Ga}}$ antisites, ${ }^{33}$ dopant atoms, ${ }^{34,35}$ heterostructures, and interfaces. ${ }^{36,37}$ It was thus demonstrated that comparison of STM images with a large variety of measurements of surface defects and calculations of density-of-states distributions allows the identification of the type of defect causing the local change in the density of states imaged by the STM. ${ }^{18}$

STM measurements revealed that as-grown Si-doped GaAs contains $V_{\mathrm{Ga}^{-}}-\mathrm{Si}_{\mathrm{Ga}}$ complexes ${ }^{16}$ which where also found by PAS. ${ }^{17}$ After annealing, an additional type of vacancy appears in highly Si-doped GaAs. ${ }^{19}$ Figure 4 shows
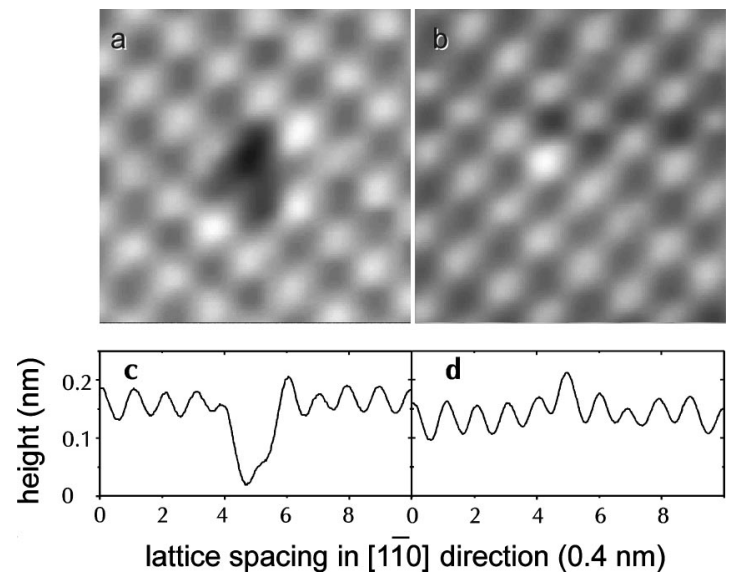

FIG. 4. Occupied (a) and empty (b) density-of-state images of the vacancy defect formed during annealing of highly Si-doped GaAs. (c) and (d) are line profiles along the atomic rows in frames (a) and (b), respectively. The images have been acquired at -2.5 and $+2.2 \mathrm{~V}(0.3 \mathrm{nA})$, respectively. The defect is identified to be a $\mathrm{Si}_{\mathrm{Ga}}$-donor-As-vacancy complex (see the text).

high-resolution constant-current images of the occupied and empty states of this defect. An occupied As dangling bond is missing, but no Ga empty dangling bond. No wide elevation or depression is detected, indicating that the defect exhibits no local band bending. Therefore, the defect is electrically neutral. The structure of the defect is very similar to that of $\mathrm{P}$ and As vacancies studied on GaP, InP, and GaAs (110) surfaces, ${ }^{29,30}$ indicating that the defect consists of an As vacancy. Since no missing Ga dangling bond is detected in the empty-state image, the defect cannot consist of a Ga vacancy. The defect exhibits an asymmetry along the atomic chains, distinctively different from previous observations of isolated vacancies. ${ }^{30}$ This indicates that the defect is not an isolated vacancy but rather a complex consisting of an As vacancy and another defect. Because $V_{\mathrm{As}}$ is expected to be negatively charged on $n$-doped GaAs $(110),{ }^{38}$ the other defect must be positively charged. In heavily Si-doped GaAs, only the $\mathrm{Si}_{\mathrm{Ga}}$ donor is positively charged. The raised empty dangling bond therefore indicates the position of a $\mathrm{Si}_{\mathrm{Ga}}$ donor. The asymmetry of the defect is the signature of a dipole character in agreement with previous observations of $\mathrm{Zn}_{\mathrm{In}}-V_{\mathrm{P}}$ (Ref. 32) and $\mathrm{Zn}_{\mathrm{Ga}}-V_{\mathrm{As}}$ (Ref. 31) complexes. Thus, the defect is assigned to a $\mathrm{Si}_{\mathrm{Ga}}$-donor-As-vacancy complex. A density of $(5.8 \pm 3.9) \times 10^{18} \mathrm{~cm}^{-3} V_{\mathrm{As}^{-}}-\mathrm{Si}_{\mathrm{Ga}}$ complexes was estimated by STM. ${ }^{19}$

On this basis we now have two types of samples: asgrown samples with $\mathrm{Ga}$-vacancy-Si $\mathrm{Sa}_{\mathrm{Ga}}$-donor complexes, and annealed samples with As-vacancy- $\mathrm{Si}_{\mathrm{Ga}}$-donor complexes. We can now use these two samples to relate the positron annihilation signals directly to the two types of vacancy defects. Thus the defect with a positron lifetime of $262 \mathrm{ps}$ is a Ga-vacancy- $\mathrm{Si}_{\mathrm{Ga}}$-donor complex, whereas the defect with the positron lifetime of about 285 ps is an As-vacancy$\mathrm{Si}_{\mathrm{Ga}}$-donor complex. These identifications are fully compatible with the above discussion of the properties estimated from PAS measurements. The concentration values are also in agreement, ${ }^{17}$ taking into account that the PAS yields a 
lower limit of the vacancy concentration in the annealed material. Based on these results we can conclude directly that positron trapping at vacancies in highly Si-doped VB GaAs and unintentionally $n$-doped $\mathrm{HB}$ GaAs is due to $V_{\mathrm{As}^{-}} \mathrm{Si}_{\mathrm{Ga}}$ complexes, and not due to isolated As vacancies or Ga vacancy-related defects. The positron annihilation signals presented above can thus be taken as reference spectra for further determinations of the types of vacancies in GaAs of otherwise unknown microscopic structure.

\section{DISCUSSION}

\section{A. Positron annihilation parameter of $V_{\mathrm{As}}-\mathrm{Si}_{\mathrm{Ga}}$ complexes-theory vs experiment}

This section will focus on a comparison of the measured positron annihilation parameters of the $V_{\mathrm{As}^{-}} \mathrm{Si}_{\mathrm{Ga}}$ complexes with theoretical calculations. The direct and independent identification of the defect complexes serves as a test ground to check the reliability of the theoretical calculations.

First, a positron lifetime of 232 ps was calculated for annihilation in defect-free GaAs. This value is slightly higher than the experimental one of $228 \pm 1 \mathrm{ps.} \mathrm{Therefore,} \mathrm{in} \mathrm{the}$ following all the calculated positron lifetimes are scaled by a factor of 228/232 to ensure direct comparability between theory and experiment. This scaling approach is adapted from Refs. 21 and 39, where it was shown to yield good agreement with experiments. The reason to use a scaling (instead of taking, e.g., the difference) is that the discrepancies between calculated and measured lifetimes are to a large degree proportional to the positron lifetime itself. This argument holds for vacancies and for the defect-free bulk, because the positron lifetimes are calculated within the socalled conventional scheme in which the electron positron correlation depends only on the electron density but not on the localization of the positron. ${ }^{21,39}$

Next we discuss the $V_{\mathrm{Ga}^{-}} \mathrm{Si}_{\mathrm{Ga}}$ complexes present in asgrown highly $\mathrm{Si}$-doped $\mathrm{GaAs}$. The experimental annihilation parameters for $V_{\mathrm{Ga}}-\mathrm{Si}_{\mathrm{Ga}}$ complexes $\left(\tau_{\mathrm{def}}=262 \pm 2 \mathrm{ps}\right.$ and $W_{\text {def }}=0.73 \pm 0.01$; Fig. 2$)$ are in close agreement with the theoretical results $\left(\tau_{\text {def }}=263 \mathrm{ps}\right.$ and $\left.W_{\text {def }}=0.72\right)$, which are calculated without taking lattice relaxation into account. This is shown in Fig. 5. The shape of the calculated momentum distribution of $V_{\mathrm{Ga}}-\mathrm{Si}_{\mathrm{Ga}}$ was also shown to be in agreement with the experiment. ${ }^{7}$ Thus the calculations provide reliable results for Ga vacancy-related defects. This is supported by recent first-principle calculations for isolated $\mathrm{Ga}$ vacancies in which lattice relaxations were fully taken into account. ${ }^{39}$ Only a weak inward relaxation was found for $V_{\mathrm{Ga}}$ resulting in a positron lifetime of $260 \mathrm{ps}$ and a $W$ parameter of $0.72,{ }^{39}$ close to the values for the unrelaxed $V_{\mathrm{Ga}}-\mathrm{Si}_{\mathrm{Ga}}$ complex calculated in the present work.

A direct identification of $V_{\mathrm{As}}-\mathrm{Si}_{\mathrm{Ga}}$ complexes now provides the opportunity to directly compare their annihilation characteristics with theoretical calculations. First the annihilation parameters for $V_{\mathrm{As}}$ or $V_{\mathrm{As}}-\mathrm{Si}_{\mathrm{Ga}}$ are calculated without taking lattice relaxation into account. The calculated positron lifetime is 263 ps for both defects. The same positron lifetime (263 ps) was calculated for the Ga-vacancy- $\mathrm{Si}_{\mathrm{Ga}}$-donor complex. This highlights the importance of additional ex-

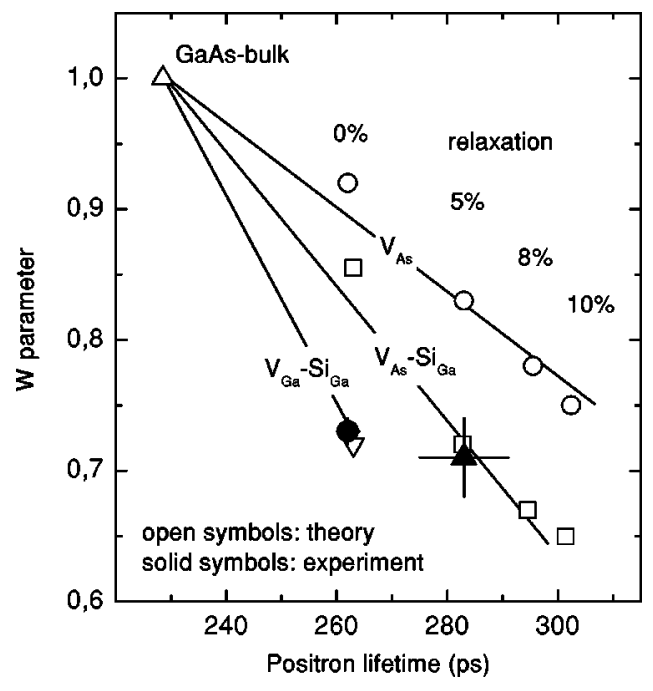

FIG. 5. $W$ parameter as a function of the average positron lifetime for defect complexes in GaAs as indicated. The lattice relaxation considered in the calculation (see text) is shown. Solid symbols are the experimental annihilation parameters for $V_{\mathrm{Ga}}-\mathrm{Si}_{\mathrm{Ga}}$ complexes $(\mathbf{O})$ and $V_{\mathrm{As}}-\mathrm{Si}_{\mathrm{Ga}}$ complexes $(\mathbf{\Lambda})$.

periments (e.g., Doppler broadening measurements) because positron lifetime measurements alone would not be able to distinguish between the different defects. The calculated $W$ parameters for $V_{\mathrm{As}}(0.92)$ and $V_{\mathrm{As}}-\mathrm{Si}_{\mathrm{Ga}}(0.86)$ are significantly higher than for $V_{\mathrm{Ga}}-\mathrm{Si}_{\mathrm{Ga}}(0.72)$, in accordance with the qualitative discussion in Sec. III A. However, the calculated values are not in agreement with the experimental results for $V_{\mathrm{As}}-\mathrm{Si}_{\mathrm{Ga}}\left(\tau_{\mathrm{def}}=283 \pm 8 \mathrm{ps}\right.$ and $W_{\mathrm{def}}=0.71 \pm 0.03$; Fig. 2). The calculated positron lifetime is lower whereas $W$ is much higher (Fig. 5). It is assumed that the calculation of positron lifetimes is not strongly influenced by the difficulties in the theoretical treatment of the annihilation momentum distribution., ${ }^{2,3}$ Therefore, the open volume of the $V_{\mathrm{As}}-\mathrm{Si}_{\mathrm{Ga}}$ complex is larger than expected, indicating an outward lattice relaxation. The same conclusion was drawn in earlier studies. ${ }^{10,12}$

Due to this discrepancy, calculations were also performed for relaxed configurations in which the distance of the four atoms neighboring the As vacancy was increased so that they move away from the center by a certain percentage of the original distance. The results are shown in Fig. 5 with the percentage of relaxation indicated. The basic idea is to find that relaxation which yields the experimental positron lifetime (280-285 ps). Then the corresponding momentum distribution can be compared with the experiment. A positron lifetime of 283 ps was calculated for an outward breathingmode relaxation of $5 \%$ for either $V_{\mathrm{As}}$ or $V_{\mathrm{As}}-\mathrm{Si}_{\mathrm{Ga}}$. The lifetime is in agreement with earlier theoretical work. ${ }^{40}$ The calculation then predicts a $W$ parameter for the $V_{\mathrm{As}}-\mathrm{Si}_{\mathrm{Ga}}$ complex $(W=0.72)$ in close agreement with the experiment ( $W=0.71$ ), whereas the calculated $W$ is much higher for the isolated As vacancy ( $W=0.83$ ) (Fig. 5). Note that the calculated annihilation parameters for the isolated As vacancy are in disagreement with the experiment even within the relatively large experimental uncertainties for the $V_{\mathrm{As}^{-}}-\mathrm{Si}_{\mathrm{Ga}}$ complex. 


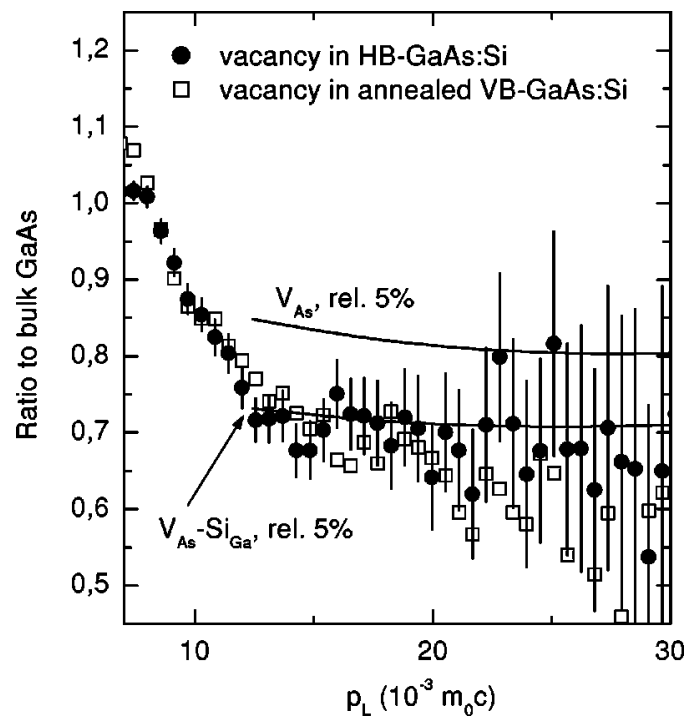

FIG. 6. High-momentum part of the annihilation momentum distribution for $V_{\mathrm{As}}-\mathrm{Si}_{\mathrm{Ga}}$ complexes in unintentionally $n$-type and annealed highly Si-doped GaAs. The data are compared with theoretical calculations for $V_{\mathrm{As}}$ and $V_{\mathrm{As}}-\mathrm{Si}_{\mathrm{Ga}}$ complexes, both relaxed outward by $5 \%$ (solid lines). The experimental data are scaled to full trapping at the defect. The theoretical results are not accurate at low momentum due to the omission of valence electrons (Ref. 3) and are therefore omitted.

The experimental annihilation momentum distribution is directly compared with the theoretical result in Fig. 6 in order to check more closely for the agreement between theory and experiment. For the comparison, the annihilation momentum distribution $f_{\text {vac }}$ of the vacancy has to be extracted from the measured momentum distribution $f_{\exp }$ shown in Fig. 3. $f_{\text {exp }}=(1-\eta) f_{\text {bulk }}+\eta f_{\text {vac }}$ is the superposition of the momentum distributions in the defect-free GaAs lattice $\left(f_{\text {bulk }}\right)$ and in the vacancy $\left(f_{\text {vac }}\right)$. The fraction $\eta$ of positrons trapped at vacancies can be determined from the positron lifetime results, and $f_{\text {bulk }}$ is known from the GaAs:Zn reference. At room temperature, $\eta$ is 0.16 in annealed highly Si-doped GaAs and 0.39 in the unintentionally $n$-doped sample. The extracted momentum distributions are shown in Fig. 6. They exhibit relatively large statistical fluctuations because $\eta$ is rather small. However, the experimental annihilation momentum distributions coincide within the statistical errors. In Fig. 6, the shape and intensity of the experimental momentum distribution are in agreement with the theoretical calculation for the $V_{\mathrm{As}}-\mathrm{Si}_{\mathrm{Ga}}$ complex imposing $5 \%$ outward relaxation, but disagree with that of the As vacancy with 5\% outward relaxation. Thus theory and experiment are in agreement, supporting further the identification of the vacancy defect as a $V_{\mathrm{As}^{-}} \mathrm{Si}_{\mathrm{Ga}}$ complex.

It was shown previously that the theory employed in the present study yields a too high intensity of the annihilation momentum distribution in GaAs due to difficulties in treating the Ga $3 d$ electrons. ${ }^{3}$ However, it might be expected that similar deviations apply for both the calculation of annihilation parameters for bulk GaAs as well as for As vacancies. This may explain the rather nice agreement of the theoretical calculations with the experiments above, since we consider here only relative changes of the momentum distribution, i.e., normalized $W$ parameters or ratio curves. Summarizing, the results of this section show that the combination of PAS experiments with theoretical calculations allows a direct identification of vacancies and vacancy complexes in both sublattices of GaAs provided the comparison is based on relative values.

\section{B. As-vacancy-related defects in GaAs}

Vacancies exhibiting a positron lifetime of around $290 \mathrm{ps}$ were earlier assigned to neutral As vacancies. ${ }^{9-12}$ The present results are based on a much more detailed microscopic identification, and show that the As vacancies form a complex with $\mathrm{Si}_{\mathrm{Ga}}$ donors. Furthermore, the complexes exhibit an outward relaxation when a positron is trapped there. Interestingly, Pöykkö et al., ${ }^{41}$ in a recent theoretical study, also found a large lattice relaxation associated with a neutral $V_{\mathrm{As}}-\mathrm{Si}_{\mathrm{Ga}}$ complex. In this work, only the displacement of the $\mathrm{Si}$ atom was considered. Two stable configurations were found, corresponding to a position of the Si atom close to the vacancy or displaced outward by about $60 \%$ along the (111) direction. We calculated the positron annihilation parameters for several such configurations with the present method. A positron lifetime of 283 ps was obtained if the $\mathrm{Si}$ atom was displaced outward by $20 \%$. The $W$ parameter was 0.74 , also in reasonable agreement with the experiment. Thus the present method of defect identification can identify a given vacancy complex, but it is not possible to discriminate between different atomic configurations, i.e., between a moderate breathing-mode outward relaxation of all surrounding atoms or a stronger outward relaxation of only one atom.

The calculated annihilation parameters agree with the experiment if the $\mathrm{Si}$ atom is displaced by only $20 \%$, in contrast to the value of $60 \%$ found to be the energetically favorable one in Ref. 41. The disagreement might be attributed to the fact that lattice relaxation of the surrounding atoms was not fully considered in Ref. 41. Nevertheless, the fact that an outward lattice relaxation is found for $V_{\mathrm{As}}-\mathrm{Si}_{\mathrm{Ga}}$ corroborates our results. In contrast, a strong inward relaxation was calculated for isolated As vacancies in the negative charge state. ${ }^{42}$ It was also predicted that the $V_{\mathrm{As}}-\mathrm{Si}_{\mathrm{Ga}}$ complex could exhibit metastability. ${ }^{41}$ However, no change of the positron annihilation parameters was observed in all samples during white light illumination at several temperatures between 30 and $300 \mathrm{~K}$.

In previous observations, a transition of the defect-related lifetime from $\sim 290$ to $\sim 260$ ps was attributed to an additional 0/- ionization level of the As vacancy. ${ }^{12}$ In the present work, this transition is not observed, neither in annealed highly Si-doped GaAs nor in unintentionally $n$-doped GaAs. The carrier concentrations in the highly Si-doped material are high enough to pin the Fermi level at the conduction band at all temperatures (see, e.g., the calculation in Ref. 10). Thus the negative charge state assigned to $V_{\mathrm{As}}$ should be observable if it exists. However, it has been shown by simulations that the 290-260-ps transition could be a pure numerical artifact due to the additional presence of negative ions acting as shallow positron traps. ${ }^{43}$ This is in agreement 
with the fact that only one ionization level for $V_{\mathrm{As}}$-related defects was detected by electrical measurements ${ }^{11}$ or optical excitation. ${ }^{44}$ However, we observe neutral defect complexes. The isolated As vacancy is therefore negatively charged in highly $n$-type GaAs, where the Fermi level is close to the conduction band. Therefore, two ionization levels $(+/ 0$ and 0/-) might be detectable by PAS for isolated As vacancies. On the other hand, recent first-principles calculations revealed a negative- $U$ behavior for the isolated As vacancy with a $+/-$ transition near midgap. ${ }^{45,46}$ Thus the neutral charge state of $V_{\mathrm{As}}$ would not be stable and only one ionization level could be detected. These calculations could provide an alternative explanation for the experimental results.

Arsenic vacancies were mostly observed by PAS in low $n$-doped GaAs, whereas in highly doped material donor $\mathrm{Ga}$ vacancy complexes (with $\tau_{\text {def }} \sim 260 \mathrm{ps}$ ) were found (see, e.g., Ref. 1 and references therein). However, in the present work no sign of positron trapping was found in weakly Si-doped GaAs $\left(n=10^{16} \mathrm{~cm}^{-3}\right.$; see Fig. 1). This particular sample was grown under As-rich conditions by the LEC method. On the other hand, vacancy-related lifetimes higher than 260 ps have also been detected in highly Si-doped horizontal Bridgman grown GaAs $\left(n \sim 2 \times 10^{18} \mathrm{~cm}^{-3}\right) .{ }^{47}$ Thus As vacancies are not necessarily present in low $n$-doped GaAs, as one might expect according to earlier work. ${ }^{10,12}$ It was commonly assumed that only Ga vacancies have a low formation energy in $n$-doped GaAs. ${ }^{48}$ However, in a recent work Chadi showed that also negatively charged As vacancies might have a low formation energy in highly $n$-doped GaAs. ${ }^{45} \mathrm{Fi}$ nally, we observed Ga-vacancy-related complexes in asgrown GaAs as well as in material annealed under high As vapor pressure. ${ }^{7}$ Both types of materials are As-rich, as is the $1 \times 10^{16} \mathrm{~cm}^{-3}$ Si-doped LEC GaAs. ${ }^{28}$ Conversely, the annealed highly Si-doped VB GaAs sample studied here contains As vacancy $\mathrm{Si}_{\mathrm{Ga}}$ donor complexes. We attribute this discrepancy to the fact that the defects present in the GaAs crystals depend critically on the exact composition. It is thus likely that the current sample has been annealed under Aspoor conditions. This interpretation is corroborated by a recent thermodynamic analysis of Hurle, who showed that horizontal Bridgman growth of GaAs occurs often under Garich conditions. ${ }^{28}$ Therefore, the unintentionally $n$-doped HB GaAs samples should represent a Ga-rich material. This is supported by the high degree of compensation observed, because it is known that growth of Si-doped GaAs under Ga- rich conditions lead to closely compensated material. ${ }^{28} \mathrm{We}$ thus infer that $V_{\mathrm{As}}$ are present preferentially in As-poor material, whereas $V_{\mathrm{Ga}}$ occurs in As-rich material, in line with the expectations from stoichiometry.

\section{SUMMARY}

In this work we demonstrated a direct identification of vacancy defects in both sublattices of GaAs by positron annihilation. This could be achieved by "calibrating" the annihilation characteristics of As vacancies with scanning tunneling microscopy measurements. In annealed highly Sidoped GaAs, we found vacancies by PAS with a positron lifetime of about 280-285 ps and a high intensity of the core annihilation momentum distribution. The annihilation characteristics are similar to those of vacancies in low $n$-doped GaAs. The intensity and shape of the annihilation momentum distribution are different from that of Ga vacancies, providing evidence that the vacancies indeed belong to the As sublattice. Using cross-sectional scanning tunneling microscopy measurements, we identified in the annealed highly Sidoped GaAs As-vacancy-Si ${ }_{\mathrm{Ga}}$-donor complexes. This identification allowed a direct comparison of the PAS data with calculations of the momentum distribution. According to the high positron lifetime, the $V_{\mathrm{As}^{-}} \mathrm{Si}_{\mathrm{Ga}}$ complexes exhibit an outward lattice relaxation. If this lattice relaxation is taken into account, the relative momentum distribution calculated for $V_{\mathrm{As}}-\mathrm{Si}_{\mathrm{Ga}}$ (but not for isolated $V_{\mathrm{As}}$ ) is in good quantitative agreement with the experiment. A similar good agreement was previously found for Ga-vacancy-related complexes. ${ }^{7}$ Therefore, the comparison of experimental PAS with theoretical calculations based on wave functions of free atoms allows a direct and unambiguous identification of vacancy defects in both sublattices of GaAs, provided normalized data are compared. We suggest that Ga vacancies are found in highly $n$-doped, arsenic-rich material. Complementarily, a Ga-rich composition favors the formation of As vacancies.

\section{ACKNOWLEDGMENTS}

We would like to thank M. J. Puska for many valuable discussions, and M. Hakala for providing the computer code for the theoretical calculation of the annihilation parameters. The authors thank the Deutsche Forschungsgemeinschaft for financial support. T.E.M.S. thanks the European Union for financial support in the form of a Marie-Curie Grant.
${ }^{1}$ R. Krause-Rehberg and H. S. Leipner, Positron Annihilation in Semiconductors (Springer-Verlag, Berlin, 1999).

${ }^{2}$ M. Alatalo, H. Kauppinen, K. Saarinen, M. J. Puska, J. Mäkinen, P. Hautojärvi, and R. M. Nieminen, Phys. Rev. B 51, 4176 (1995).

${ }^{3}$ M. Alatalo, B. Barbiellini, M. Hakala, H. Kauppinen, T. Korhonen, M. J. Puska, K. Saarinen, P. Hautojärvi, and R. M. Nieminen, Phys. Rev. B 54, 2397 (1996).

${ }^{4}$ P. Asoka-Kumar, M. Alatalo, V. J. Ghosh, A. C. Kruseman, B. Nielsen, and K. G. Lynn, Phys. Rev. Lett. 77, 2097 (1996).
${ }^{5}$ K. G. Lynn and A. N. Goland, Solid State Commun. 18, 1549 (1976).

${ }^{6}$ K. G. Lynn, J. R. MacDonald, R. A. Boie, L. C. Feldman, J. D. Gabbe, M. F. Robbins, E. Bonderup, and J. Golovchenko, Phys. Rev. Lett. 38, 241 (1977).

${ }^{7}$ J. Gebauer, M. Lausmann, T. E. M. Staab, R. Krause-Rehberg, M. Hakala, and M. J. Puska, Phys. Rev. B 60, 1464 (1999).

${ }^{8}$ B. Barbiellini, M. Hakala, M. J. Puska, and R. M. Nieminen, Phys. Rev. B 56, 7136 (1997).

${ }^{9}$ G. Dlubek and R. Krause, Phys. Status Solidi A 102, 443 (1987). 
${ }^{10}$ C. Corbel, M. Stucky, P. Hautojärvi, K. Saarinen, and P. Moser, Phys. Rev. B 38, 8192 (1988).

${ }^{11}$ R. Krause, A. Polity, G. Dlubek, K. Friedland, R. Rentzsch, W. Siegel, and G. Kühnel, in Positron Annihilation, edited by L. Dorikens-Vanpraet, M. Dorikens, and D. Segers (World Scientific, Singapore, 1989), p. 721.

${ }^{12}$ K. Saarinen, P. Hautojärvi, P. Lanki, and C. Corbel, Phys. Rev. B 44, 10585 (1991).

${ }^{13}$ R. Ambigapathy, A. A. Manuel, P. Hautojärvi, K. Saarinen, and C. Corbel, Phys. Rev. B 50, 2188 (1994).

${ }^{14}$ J. P. Peng, K. G. Lynn, M. T. Umlor, D. J. Keeble, and D. R. Harshman, Phys. Rev. B 50, 11247 (1994).

${ }^{15}$ L. Gilgien, G. Galli, F. Gygi, and R. Car, Phys. Rev. Lett. 72, 3214 (1994).

${ }^{16}$ C. Domke, Ph. Ebert, M. Heinrich, and K. Urban, Phys. Rev. B 54, 10288 (1996).

${ }^{17}$ J. Gebauer, R. Krause-Rehberg, C. Domke, Ph. Ebert, and K. Urban, Phys. Rev. Lett. 78, 3334 (1997).

${ }^{18} \mathrm{Ph}$. Ebert, Surf. Sci. Rep. 33, 121 (1999).

${ }^{19}$ C. Domke, Ph. Ebert, and K. Urban, Phys. Rev. B 57, 4482 (1998)

${ }^{20}$ J. Gebauer, R. Krause-Rehberg, S. Eichler, and F. Börner, Appl. Surf. Sci. 149, 110 (1999).

${ }^{21}$ B. Barbiellini, M. J. Puska, T. Korhonen, A. Harju, T. Torsti, and R. M. Nieminen, Phys. Rev. B 53, 16201 (1996).

${ }^{22}$ K. Saarinen, P. Hautojärvi, A. Vehanen, R. Krause, and G. Dlubek, Phys. Rev. B 39, 5287 (1989).

${ }^{23}$ M. J. Puska, C. Corbel, and R. M. Nieminen, Phys. Rev. B 41, 9980 (1990).

${ }^{24}$ R. Krause-Rehberg and H. S. Leipner, Appl. Phys. A: Mater. Sci. Process. 64, 457 (1997).

${ }^{25}$ M. J. Puska, J. Phys.: Condens. Matter 1, 7347 (1989).

${ }^{26}$ R. W. Jansen and O. F. Sankey, Phys. Rev. B 39, 3192 (1989).

${ }^{27}$ J. Gebauer, F. Börner, R. Krause-Rehberg, T. E. M. Staab, W. Bauer-Kugelmann, G. Kögel, W. Triftshäuser, P. Specht, R. C. Lutz, E. R. Weber, and M. Luysberg, J. Appl. Phys. 87, 8368 (2000).
${ }^{28}$ D. T. J. Hurle, J. Appl. Phys. 58, R6957 (1999).

${ }^{29}$ G. Lengel, J. Harper, M. Weimer, J. Gryko, and R. E. Allen, Phys. Rev. Lett. 72, 836 (1994).

${ }^{30} \mathrm{Ph}$. Ebert, K. Urban, and M. G. Lagally, Phys. Rev. Lett. 72, 840 (1994).

${ }^{31}$ K.-J. Chao, A. R. Smith, and C.-K. Shih, Phys. Rev. B 53, 6935 (1996).

${ }^{32} \mathrm{Ph}$. Ebert, M. Heinrich, M. Simon, C. Domke, K. Urban, C. K. Shih, M. B. Webb, and M. G. Lagally, Phys. Rev. B 53, 4580 (1996).

${ }^{33}$ R. M. Feenstra, J. M. Woodall, and G. D. Pettit, Phys. Rev. Lett. 71, 1176 (1993).

${ }^{34}$ J. F. Zheng, X. Liu, N. Newman, E. R. Weber, D. F. Ogletree, and M. Salmeron, Phys. Rev. Lett. 72, 1490 (1994).

${ }^{35}$ M. B. Johnson, O. Albrektsen, R. M. Feenstra, and H. W. M. Salemink, Appl. Phys. Lett. 63, 2923 (1993).

${ }^{36}$ M. B. Johnson, P. M. Koenraad, W. C. van der Vleuten, H. W. M. Salemink, and J. H. Wolter, Phys. Rev. Lett. 75, 1606 (1995).

${ }^{37}$ J. F. Zheng, J. D. Walker, M. B. Salmeron, and E. R. Weber, Phys. Rev. Lett. 72, 2414 (1994).

${ }^{38}$ S. B. Zhang and A. Zunger, Phys. Rev. Lett. 77, 119 (1996).

${ }^{39}$ M. J. Puska, A. P. Seitsonen, and R. M. Nieminen, Phys. Rev. B 52, 10947 (1995).

${ }^{40}$ M. J. Puska and C. Corbel, Phys. Rev. B 38, 9874 (1988).

${ }^{41}$ S. Pöykkö, M. J. Puska, M. Alatalo, and R. M. Nieminen, Phys. Rev. B 54, 7909 (1996).

${ }^{42}$ K. Laasonen, R. M. Nieminen, and M. J. Puska, Phys. Rev. B 45, 4122 (1992).

${ }^{43}$ B. Somieski, T. E. M. Staab, and R. Krause-Rehberg, Nucl. Instrum. Methods Phys. Res. A 381, 128 (1996).

${ }^{44}$ K. Saarinen, S. Kuisma, P. Hautojärvi, C. Corbel, and C. LeBerre, Phys. Rev. Lett. 70, 2794 (1993).

${ }^{45}$ D. J. Chadi, Mater. Sci. Forum 258-263, 1321 (1997).

${ }^{46}$ S. Pöykkö, M. J. Puska, and R. M. Nieminen, Phys. Rev. B 53, 3813 (1996).

${ }^{47}$ S. Fujii, A. Uedono, and S. Tanigawa, Hyperfine Interact. 79, 719 (1993).

${ }^{48}$ S. B. Zhang and J. E. Northrup, Phys. Rev. Lett. 67, 2339 (1991). 